\title{
Extracellular matrix metalloproteinase inducer and matrix metalloproteinase- 2 overexpression is associated with loss of hormone receptor expression and poor prognosis in endometrial cancer
}

\author{
YUAN YUAN $^{1}$, NING SHEN ${ }^{2}$, SHU-YAN YANG $^{1}$, LING ZHAO $^{1}$ and YONG-MEI GUAN ${ }^{3}$ \\ ${ }^{1}$ Department of Gynecology, The Fourth Affiliated Hospital of Harbin Medical University, Harbin, Heilongjiang 150001; \\ ${ }^{2}$ Department of Pathophysiology, Heilongjiang University of Chinese Medicine, Harbin, Heilongjiang 150040; \\ ${ }^{3}$ Department of Gynecology, The First Affiliated Hospital of Harbin Medical University, Harbin, \\ Heilongjiang 150001, P.R. China
}

Received May 11, 2014; Accepted February 2, 2015

DOI: $10.3892 / \mathrm{ol} .2015 .3177$

\begin{abstract}
Extracellular matrix metalloproteinase inducer (CD147) and matrix metalloproteinase-2 (MMP-2) have been documented in various malignancies. CD147 is a member of the immunoglobulin superfamily, which promotes the production and release of MMPs in mesenchymal cells and tumor cells. MMP-2 has been extensively studied and is considered to be particularly important in cancer invasion and metastasis. However, studies investigating the expression and prognostic value of CD147 in endometrioid endometrial carcinoma (EEC) are limited. The present study analyzed the expression of CD147 and MMP-2 by immunohistochemistry in endometrial tissue samples from 107 patients with EEC and 30 patients with benign uterus myoma. The association between CD147 and MMP-2 expression and clinicopathological characteristics was evaluated. The results showed that the overexpression of MMP-2 was significantly associated with International Federation of Gynecology and Obstetrics stage $(\mathrm{P}=0.007)$, depth of invasion $(\mathrm{P}=0.037)$ and reduced expression of progesterone receptor $(\mathrm{P}=0.005)$. Kaplan-Meier analyses indicated that $\mathrm{CD} 147$ overexpression alone $(\mathrm{P}<0.05$ for disease-specific survival) or in combination with MMP-2 $(\mathrm{P}<0.001$ for disease-specific survival) was correlated with adverse prognosis in EEC patients. Multivariate analysis revealed that the combined overexpression of CD147 and MMP-2 was an
\end{abstract}

Correspondence to: Dr Yong-Mei Guan, Department of Gynecology, The First Affiliated Hospital of Harbin Medical University, 23 Youzheng Street, Harbin, Heilongjiang 150001, P.R. China

E-mail: harbinmu03@163.com

Key words: endometrioid endometrial cancer, extracellular matrix metalloproteinase inducer, matrix metalloproteinase-2, immunohistochemistry, prognosis independent prognostic factor for disease-specific survival (hazard ratio=5.141, $\mathrm{P}=0.001$ ) in EEC patients. CD147 and MMP-2 overexpression was positively correlated with aggressive phenotypic features in EEC, however it was negatively correlated with hormone receptor expression. The combination of CD147 and MMP-2 overexpression in EEC further distinguished a subgroup of patients with poor prognosis. Thus, the results of present study indicate that the co-expression of CD147 and MMP-2 may be an independent prognostic factor in EEC patients.

\section{Introduction}

Endometrial carcinoma (EC) is one of the most common gynecological malignancies worldwide (1), accounting for $20-30 \%$ of all gynecological tumors. Endometrioid endometrial cancer (EEC) is the most common type of EC, which accounts for $80-85 \%$ cases of EC. It is frequently diagnosed at an early stage and responds well to surgical treatment, which is the primary treatment option, with a five-year survival rate of $\sim 80 \%$. Although the overall survival rate of patients with this tumor has markedly improved since the 1980's, due to earlier detection and treatment, the prognosis remains poor in cases of advanced or recurrent EC (2). Several clinicopathological factors have been demonstrated to be associated with prognosis in cases of EC, including histological grade, International Federation of Gynecology and Obstetrics (FIGO) stage and lymph node metastasis $(2,3)$. Certain markers have been shown to be of prognostic significance, however, the molecular mechanisms of carcinogenesis and progression of this cancer remain unclear.

Extracellular matrix metalloproteinase inducer (CD147) is a highly glycosylated transmembrane protein, and a member of the immunoglobulin superfamily (4). It has been reported to be expressed on the surface of tumor cells, and may promote the production or release of matrix metalloproteinases (MMPs) in mesenchymal cells and tumor cells (5). MMPs degrade the extracellular matrix around tumor cells and therefore facilitate 
invasion and metastasis in cancer $(3,6,7)$. MMP-2 has been extensively studied and is particularly important in cancer invasion and metastasis. Studies have shown that CD147 is overexpressed in a number of human cancer types, including gastric carcinoma, breast cancer, prostate cancer and cervical cancer (8-10), and the overexpression of CD147 is associated with an aggressive cancer phenotype and poor prognosis for the patient. Additionally, the overexpression of CD147 in malignant cells is associated with metastatic and invasive ability, and sensitivity to growth inhibitory signals (11). Furthermore, CD147 is frequently expressed in primary tumors and in micrometastatic cancer cells. These results suggest that CD147 is a crucial contributor to tumor progression. In gallbladder carcinoma, CD147/MMP-2 co-expression has been shown to be an independent prognostic indicator, and may be a more reliable predictor of outcome compared with individual expression (12). However, the prognostic value of these markers in EC is yet to be established.

The expression of steroid hormone receptors [progesterone receptor (PR) or estrogen receptor (ER)] in patients with EC has previously been reported to be a good prognostic indicator $(13,14)$. The association between ER and PR expression in EC and certain prognostic variables, including tumor FIGO stage, depth of myometrial invasion, histological grade and survival, has been established (14). In addition, steroid hormones, particularly estrogen, have been identified as important molecules in EC pathogenesis; elevated estrogen stimulation is associated with carcinogenesis and malignant transformation of benign endometrium in EEC (15). A decrease in ER and PR expression is frequently observed in EC, and may also contribute to carcinogenesis of the endometrium (8).

This study assessed the co-expression of CD147 and MMP-2, and the association of these molecules with clinicopathological characteristics, and with ER and PR expression in cases of EC, in order to evaluate their involvement in tumor progression.

\section{Materials and methods}

Patients and tissue samples. Specimens of endometrial tissue were collected from 30 control subjects and 107 patients treated for EEC between September 2002 and March 2005 (age range, 35-76 years; FIGO stage, I-V). The control subjects were females undergoing hysterectomy for the treatment of uterus myoma at the Department of Gynecology, Second Affiliated Hospital of Harbin Medical University (Harbin, China). All primary EEC patients who received surgery were included in the study. Patients who had previously undergone radiotherapy, chemotherapy or immunotherapy were excluded. The pathological diagnosis was conducted preoperatively and confirmed postoperatively by histopathological examination. The study was approved by the Ethics Committee of The Fourth Affiliated Hospital of Harbin Medical University (Harbin, China), and written informed consent was obtained from all patients.

The clinicopathological characteristics of the included EEC cases are summarized in Table I. All specimens were anonymized and handled according to ethical and legal standards. The end date of the follow-up study for analysis was March 31, 2009, and the median duration of follow-up was 72 months (range, 9-78 months). Of the 110 EEC patients, three were lost to follow-up. Either patients who were alive at final follow-up or patients succumbed to conditions other than EEC were excluded at the date of last follow-up. The staging of tumors was performed according to FIGO criteria $(16,17)$.

Immunohistochemistry. The immunohistochemical staining of paraffin-embedded tissue samples was performed according to a previously described protocol (18). Samples were cut using a microtome into sections of 5-10 $\mu \mathrm{m}$ in thickness. Hematoxylin and eosin staining was used to confirm the presence of tumor tissue prior to immunohistochemical staining. Sections were deparaffinized and incubated with CD147 monoclonal antibody (dilution, 1:75; cat. no. ZM-0445), MMP-2 monoclonal antibody (dilution, 1:75; cat. no. ZA-0331), PR monoclonal antibody (dilution, 1:100; cat. no. ZA-0102) or ER polyclonal antibody (dilution, 1:150; cat. no. ZA-0255) (Santa Cruz Biotechnology, Santa Cruz, CA, USA) for $30 \mathrm{~min}$. The slides were rinsed with phosphate-buffered saline (PBS), followed by a 30-min incubation with a secondary antibody (dilution, 1:75; cat. no. ZDR-5119; Beijing Zhongshan Jinqiao Biotechnology Co., Ltd., Beijing, China) and a further wash with PBS. Sections were subsequently incubated with a ready-to-use DAB solution for 5-15 min until a brown color became visible, and the reaction was discontinued by washing in running water.

Immunohistochemical staining evaluation. Tissue sections were independently evaluated by two gynecological pathologists who were blinded to the identity and clinical status of each patient. The immunohistochemical expression was semi-quantitatively measured, as described by Zhong et al (9), by assessing the percentage and staining intensity of stained tumor cells. Samples were scored from 0-3 based on the percentage of cells exhibiting positive staining as follows: 0 points, $0-5 \% ; 1$ point, $6-50 \% ; 2$ points, $51-75 \%$; and 3 points, $>75 \%$. Staining intensity was scored as follows: 2 points, weak intensity; 3 points, moderate intensity; and 4 points, strong intensity. An overall score index (SI) was calculated for each sample by multiplying the intensity score by the percentage score. Tumors were categorized into one of three groups based on their SI: group 1, negative or weak expression (SI, 0-4); group 2, moderate expression (SI, 5-8), and group 3, strong expression (SI, 9-12).

Statistical analysis. Data were analyzed using SPSS version 16.0 for Windows (SPSS, Inc., Chicago, IL, USA). The categorical variables were analyzed using Pearson's $\chi^{2}$ test, and Spearman's correlation test was used for the rank data analysis. Survival curves were calculated according to the Kaplan-Meier method and evaluated using the log-rank test. Cox regression based on a proportional hazard model was performed for multivariate analysis of prognostic predictors. $\mathrm{P}<0.05$ was considered to indicate a statistically significant difference.

\section{Results}

Expression of CD147 and MMP-2 in EEC. Immunohistochemical evaluation indicated that, of the 30 control 
Table I. Association analyses between the expression levels of CD147 and MMP-2 and the clinicopathological characteristics of endometrioid endometrial cancer.

\begin{tabular}{|c|c|c|c|c|c|c|c|c|c|}
\hline \multirow{2}{*}{$\begin{array}{l}\text { Clinicopathological } \\
\text { variable }\end{array}$} & \multirow{2}{*}{$\begin{array}{c}\text { Patients, } \\
\text { n }\end{array}$} & \multicolumn{4}{|c|}{ CD147 expression } & \multicolumn{4}{|c|}{ MMP-2 expression } \\
\hline & & Low, $\mathrm{n}$ & High, $n$ & $\chi^{2}$ & P-value & Low, $\mathrm{n}$ & High, $n$ & $\chi^{2}$ & P-value \\
\hline All cases & 107 & 59 & 48 & & & 58 & 49 & & \\
\hline Age, years & & & & 6.24 & 0.009 & & & 9.67 & 0.002 \\
\hline$>60$ & 46 & 19 & 27 & & & 17 & 29 & & \\
\hline$\leq 60$ & 61 & 40 & 21 & & & 41 & 20 & & \\
\hline FIGO stage & & & & 0.445 & 0.505 & & & 7.316 & 0.007 \\
\hline Low (I/II) & 77 & 44 & 33 & & & 48 & 29 & & \\
\hline High (III/IV) & 30 & 15 & 15 & & & 10 & 20 & & \\
\hline Histological grade & & & & 1.611 & 0.204 & & & 6.669 & 0.010 \\
\hline Low (G1 or 2) & 84 & 49 & 35 & & & 51 & 33 & & \\
\hline High (G3) & 23 & 10 & 13 & & & 7 & 16 & & \\
\hline Lymph node metastasis & & & & 6.801 & 0.009 & & & 2.668 & 0.102 \\
\hline Negative & 74 & 47 & 27 & & & 44 & 30 & & \\
\hline Positive & 33 & 12 & 21 & & & 14 & 19 & & \\
\hline Depth of myometrial invasion & & & & 3.394 & 0.065 & & & 4.348 & 0.037 \\
\hline$<50 \%$ & 66 & 41 & 25 & & & 41 & 25 & & \\
\hline$\geq 50 \%$ & 41 & 18 & 23 & & & 17 & 24 & & \\
\hline ER expression & & & & 10.025 & 0.002 & & & 3.128 & 0.077 \\
\hline Low & 60 & 25 & 35 & & & 28 & 32 & & \\
\hline High & 47 & 34 & 13 & & & 30 & 17 & & \\
\hline PR expression & & & & 2.155 & 0.142 & & & 7.962 & 0.005 \\
\hline Low & 54 & 26 & 28 & & & 22 & 32 & & \\
\hline High & 53 & 33 & 20 & & & 36 & 17 & & \\
\hline
\end{tabular}

FIGO, International Federation of Gynecology and Obstetrics; ER, estrogen receptor; PR, progesterone receptor; CD147, extracellular matrix metalloproteinase inducer; MMP-2, matrix metalloproteinase-2.

specimens, 27 (90\%) exhibited low (negative or weak) CD147 expression levels (SI, 0-8; Fig. 1C) and 26 (86.67\%) exhibited low MMP-2 expression (SI, 0-8; Fig. 1D). Of the 107 EEC specimens, 48 (44.9\%) exhibited high CD147 expression (SI, 9-12; Fig. 1A) and 59 (55.1\%) exhibited low CD147 expression (SI, 0-8); 49 samples (45.8\%) exhibited high MMP-2 expression (SI, 9-12; Fig. 1B) and 58 (54.2\%) exhibited low MMP-2 expression (SI, 0-8). Notably, 32 samples (29.9\%) exhibited both high MMP-2 expression and high CD147 expression (SI, 9-12).

Association between the expression of CD147 and MMP-2 and the clinicopathological characteristics of EEC. As shown in Table I, no correlation was observed between the expression of CD147 and MMP-2 and the age of the patient. MMP-2 was overexpressed in $66.6 \%$ of patients with high FIGO stage disease, compared with $37.7 \%$, of patients with low-stage disease; this suggests that the expression of MMP-2 protein is associated with FIGO stage $(\mathrm{P}=0.007)$. However, no significant difference in CD147 expression was observed between high and low FIGO stage samples. MMP-2 expression was also significantly associated with depth of myometrial invasion $(\mathrm{P}=0.037)$, and the overexpression of
CD147 protein was associated with lymph node metastasis $(\mathrm{P}=0.009)$.

Correlation between the expression of CD147 and MMP-2. Pearson's $\chi^{2}$ test demonstrated that the elevated expression level of CD147 was highly correlated with expression of MMP-2 ( $\mathrm{P}=0.02)$.

Clinical outcome and CD147 and MMP-2 expression. The association between clinical characteristics and the disease specific survival (DSS) of EEC patients was evaluated by univariate analysis. As shown in Table II, advanced age ( $>60$ years) was significantly associated with an increased mortality rate from EEC $(\mathrm{P}=0.025)$. A myometrial invasion depth of $\geq 50 \%$, high tumor grade and lymph node metastasis also predicted a shorter DSS $(\mathrm{P}<0.001)$.

The association between the expression of CD147 and MMP-2 and aggressive pathological characteristics in EEC patients was also evaluated. The expression of CD147 and MMP-2 in these patients was categorized into four groups: CD147 and MMP-2 double negative; CD147 negative and MMP-2 positive; CD147 positive and MMP-2 negative; and CD147 and MMP-2 double positive. A pair-wise comparison 
A
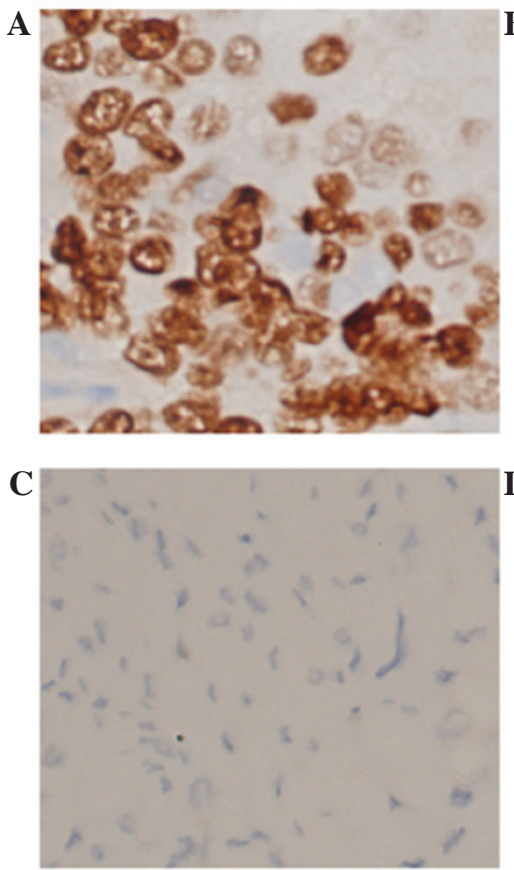
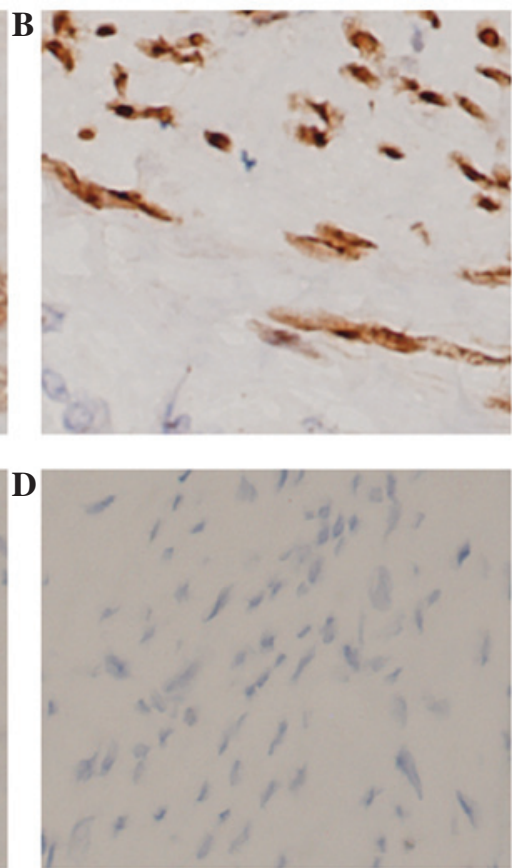

Figure 1. The typical expression pattern of CD147 and MMP-2 in normal endometrium and EEC tissues, evaluated by immunohistochemical staining (original magnification, x200). (A) Strong positive expression of CD147 in the cell membrane in EEC tissues. (B) Strong positive expression of MMP-2 in the cytoplasm in EEC tissues. (C) Negative staining of CD147 in normal endometrial tissue. (D) Negative staining of MMP-2 in normal endometrial tissue. EEC, endometrioid endometrial carcinoma; CD147, extracellular matrix metalloproteinase inducer; MMP-2, matrix metalloproteinase-2.
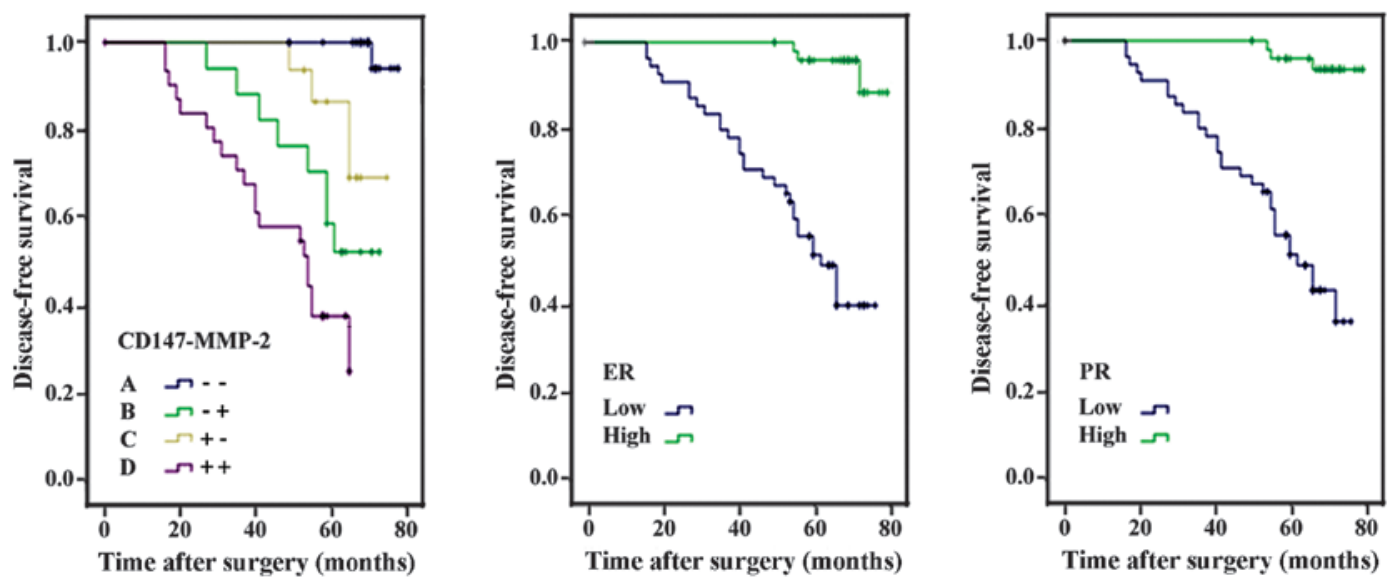

Figure 2. Kaplan-Meier survival curves for CD147 and MMP-2 (Panel 1), ER (Panel 2) and PR (Panel 3) expression in patients with EEC. (A) CD147 and MMP-2 double negative expression; (B) CD147 negative, MMP-2 positive expression; (C) CD147 positive, MMP-2 negative expression; and (D) CD147 and MMP-2 double positive expression. Survival for patients with B subtypes was significantly increased compared with all other subtypes $(\mathrm{P}<0.01)$. EEC, endometrioid endometrial carcinoma; CD147, extracellular matrix metalloproteinase inducer; MMP-2, matrix metalloproteinase-2; ER, estrogen receptor; PR, progesterone receptor.

analysis revealed that the DSS of CD147+/MMP-2+ patients was significantly shorter compared with each of the other three groups $(\mathrm{P}<0.01)$. Cox regression analysis showed that the combined expression of CD147+/MMP-2+ and CD147-/MMP-2- is an independent prognostic indicator for EEC patients (Table III; $\mathrm{P}<0.01$ ). The Kaplan-Meier curves for CD147/MMP-2 co-expression in the EEC patients are shown in Fig. 2.

ER and PR were demonstrated to be highly expressed in $43.9 \%$ and $49.5 \%$ of EEC samples, respectively (Fig. 1). CD147 overexpression was detected in $58.3 \%$ of tumors with low ER expression, compared with $27.7 \%$ of tumors with high ER expression; this difference was statistically significant $(\mathrm{P}=0.002)$. Similarly, MMP-2 overexpression was detected in 59.3\% of tumors with low PR expression compared with $32.1 \%$ of tumors with high PR expression $(\mathrm{P}=0.005)$.

\section{Discussion}

The present study assessed the overexpression of CD147 and MMP-2 in EEC using immunohistochemistry, and demonstrated that overexpression of MMP-2 was significantly associated with depth of myometrial invasion, histological grade and FIGO stage. Furthermore, the overexpression of 
Table II. Univariate survival analysis of DSS in patients with endometrioid endometrial cancer $(\mathrm{n}=107)$.

\begin{tabular}{|c|c|c|c|c|c|c|c|}
\hline \multirow[b]{3}{*}{ Variable } & \multirow[b]{3}{*}{$\begin{array}{c}\text { Patients, } \\
\mathrm{n}\end{array}$} & \multirow[b]{3}{*}{ Estimate } & \multicolumn{3}{|c|}{ DSS } & \multirow[b]{3}{*}{$\chi^{2 \mathrm{a}}$} & \multirow[b]{3}{*}{ P-value } \\
\hline & & & \multirow[b]{2}{*}{$\begin{array}{l}\text { Standard } \\
\text { error }\end{array}$} & \multicolumn{2}{|c|}{$95 \% \mathrm{CI}$} & & \\
\hline & & & & $\begin{array}{l}\text { Lower } \\
\text { bound }\end{array}$ & $\begin{array}{l}\text { Upper } \\
\text { bound }\end{array}$ & & \\
\hline Age, years & & & & & & 5.044 & 0.025 \\
\hline$\leq 60$ & 61 & 65.241 & 1.178 & 62.932 & 67.550 & & \\
\hline$>60$ & 46 & 63.722 & 1.118 & 61.532 & 65.913 & & \\
\hline FIGO stage & & & & & & 0.062 & 0.804 \\
\hline $\mathrm{I} / \mathrm{II}$ & 77 & 65.108 & 0.899 & 63.347 & 66.869 & & \\
\hline III/IV & 30 & 62.846 & 2.380 & 58.181 & 67.510 & & \\
\hline Histological grade & & & & & & 23.728 & $<0.001$ \\
\hline 1 or 2 & 84 & 65.707 & 0.875 & 63.993 & 67.422 & & \\
\hline 3 & 23 & 56.300 & 1.542 & 53.277 & 59.323 & & \\
\hline Depth of myometrial invasion & & & & & & 14.225 & $<0.001$ \\
\hline$<50 \%$ & 66 & 66.279 & 0.950 & 64.418 & 68.140 & & \\
\hline$\geq 50 \%$ & 41 & 60.508 & 1.405 & 57.753 & 63.262 & & \\
\hline Lymph node status & & & & & & 16.602 & $<0.001$ \\
\hline Negative & 74 & 66.554 & 0.745 & 65.094 & 68.014 & & \\
\hline Positive & 33 & 56.608 & 2.173 & 52.350 & 60.866 & & \\
\hline ER expression & & & & & & 4.765 & 0.029 \\
\hline Low & 60 & 63.672 & 1.100 & 61.516 & 65.827 & & \\
\hline High & 47 & 65.686 & 1.297 & 63.144 & 68.229 & & \\
\hline PR expression & & & & & & 7.981 & 0.005 \\
\hline Low & 54 & 62.381 & 1.250 & 59.931 & 64.830 & & \\
\hline High & 53 & 66.591 & 1.093 & 64.449 & 68.733 & & \\
\hline CD147/MMP-2 & & & & & & 22.230 & $<0.001$ \\
\hline CD147-/MMP-2- & 42 & 68.051 & 1.128 & 65.840 & 70.263 & & \\
\hline CD147-/MMP-2+ & 17 & 65.692 & 1.452 & 62.847 & 68.538 & & \\
\hline CD147+/MMP-2- & 16 & 61.978 & 1.381 & 59.270 & 64.685 & & \\
\hline CD147+/MMP-2+ & 32 & 58.188 & 2.419 & 53.446 & 62.929 & & \\
\hline
\end{tabular}

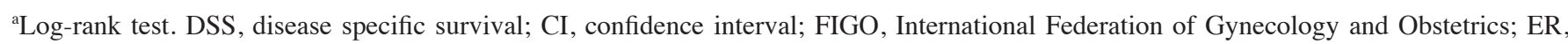
estrogen receptor; PR, progesterone receptor.

CD147 and MMP-2 served as an independent prognostic factor for poor DSS in advanced EEC patients.

The cell surface protein CD147 is located on the surface of tumor cells and functions as an upstream modulator of MMP-2, which is found to be highly expressed in a number of cancer types, including gallbladder carcinoma, thyroid carcinoma, lung squamous cell carcinoma and laryngeal carcinoma (19-23). The overexpression of CD147 has been reported in the majority of invasive carcinomas, including thyroid carcinoma and laryngeal carcinoma, indicating a that this protein is important in neoplastic progression $(24,25)$. Yu et al (10) found that overexpression of CD147 was correlated with a highly-invasive phenotype in squamous cell carcinoma of the uterine cervix. In addition, Zhao et al (26) identified a significant association between CD147 overexpression and poor disease-free and overall survival rates in patients with prostate cancer, indicating that CD147 is an independent risk factor for this type of cancer. In line with previous studies, the results of the present study showed a significantly increased rate of CD147 overexpression in endometrial tissue samples from patients with EEC, compared with that in benign tissue samples. Additionally, a significant positive correlation between the expression of CD147/MMP-2 and clinicopathological characteristics was demonstrated: CD147 overexpression was associated with lymph node metastasis, while MMP-2 overexpression was associated with high FIGO stage, high histological grade and increased myometrial invasion ( $\geq 50 \%)$. Furthermore, the expression of CD147 appears to be correlated with the expression of MMP-2. These results suggest that overexpression of CD147 and MMP-2 may be important in EEC carcinogenesis and progression.

Several studies have suggested that CD147 stimulates MMP-2 production in addition to promoting tumor angiogenesis by regulating the expression of vascular endothelial 
Table III. Multivariate survival analysis using Cox proportional hazards model $(n=107)$.

95\% CI for $\operatorname{Exp}(\mathrm{B})$

\begin{tabular}{lcccccccr} 
& B & SE & Wald & Df & Sig. & Exp (B) & Lower & Upper \\
\hline Histological grade & 0.695 & 0.203 & 11.718 & 1 & 0.001 & 2.004 & 1.346 & 2.984 \\
Lymph node status & 1.080 & 0.334 & 10.466 & 1 & 0.001 & 2.945 & 1.531 & 5.667 \\
CD147/MMP-2 : - - & & & 15.821 & 3 & 0.001 & & & \\
CD147/MMP-2(1): - & 0.502 & 0.329 & 2.327 & 1 & 0.127 & 1.652 & 0.867 & 3.148 \\
CD147/MMP-2(2): + -1.007 & 0.325 & 9.615 & 1 & 0.002 & 2.736 & 1.448 & 5.170 \\
CD147/MMP-2(3): + + & 1.032 & 0.309 & 11.164 & 1 & 0.001 & 2.807 & 1.532 & 5.141 \\
\hline
\end{tabular}

Cox regression test: Forward Stepwise (Wald). CI, confidence interval.

growth factor (VEGF) (14,27). Downregulation of CD147 by specific siRNA has been shown to inhibit the secretion of MMPs and VEGF, and suppress the invasion of human glioblastoma cells (28). CD147 also regulates proliferation in malignant cells through the activation of mitogen activated protein kinases (MAPKs), including ERK1/2 and p38 (29). It may also contribute to the multi-drug resistance (MDR) phenotype through elevating ErbB2 signaling and cell survival pathways (30). Taken together, these findings imply that CD147 may be a therapeutic target to overcome metastasis and MDR in cancer. The present study demonstrated that the overexpression of CD147 and MMP-2 are associated with metastasis and invasion activities, respectively, and may be a reliable indicator of poor prognosis. However, the specific mechanism by which overexpression of CD147 and MMP-2 affect cancer progression is unclear and requires further investigation.

FIGO stage is a reliable prognostic factor, however, it cannot perfectly predict the outcome for every individual (31). With the development of molecular techniques, certain biological molecules, including CD39 and CD73, have been shown to be important in diagnosis, prognosis, and treatment of human EC (32). Previous studies demonstrated the potential value of CD147 in the prognosis of EC, but its prognostic power is insufficient $(33,34)$. Therefore, it is necessary to evaluate a combination of factors to improve the sensitivity and specificity of cancer prognostic prediction. In the present study, the predictive value of CD147/MMP-2 co-expression in the prognosis of EEC was analyzed. The data indicated that patients with high CD147+/MMP-2+ expression had significantly poorer disease free survival compared with patients with CD147-/MMP-2- expression, based on log-rank tests and Kaplan-Meier analysis. Furthermore, multivariate analysis revealed that CD147 and MMP-2 double negative expression, and CD147 and MMP-2 double positive expression were independent prognostic indicators of survival rate in EEC, and may serve as potential biomarkers for the prediction of prognosis in EEC. The combined evaluation of CD147 and MMP-2 expression may be more convenient and efficient compared with the current standard prognostic methods.

High levels of ER and PR are considered reliable indicators for a favorable clinical outcomes, and are associated with lower tumor grades, lower incidence of lymph node metastases and less myometrial invasion (35). Hormone therapies are used to treat patients with recurrent or advanced endometrial cancers, and the best responses are observed in well-differentiated and steroid hormone receptor positive tumors (36). In the present study, low expression of ER and PR was significantly associated with a shorter DSS, and the expression of CD147 and MMP-2 was inversely correlated with levels of ER and PR, respectively, in EEC tissues. Additionally, CD147 and MMP-2 co-expression defined a subgroup of patients with an aggressive pathological phenotype and poor prognosis. Taken together, these findings suggest that poorly differentiated endometrial cancers may be associated with steroid hormone deficiency.

In conclusion, the results of the current study suggest that co-expression of CD147 and MMP-2 may be an independent prognostic factor for DSS in patients with EEC. This may be useful in the prediction of prognosis in cases of EEC, and also aid in the selection of treatment for EEC patients. Further investigation is required to develop this method and assess its value.

\section{References}

1. Cancer statistics. JAMA 310: 982, 2013.

2. Fowler W and Mutch D: Management of endometrial cancer. Womens Health (Lond Engl) 4: 479-489, 2008.

3. Millimaggi D, Mari M, D'Ascenzo S, et al: Tumor vesicle-associated CD147 modulates the angiogenic capability of endothelial cells. Neoplasia 9: 349-357, 2007.

4. Sidhu SS, Mengistab AT, Tauscher AN, LaVail J and Basbaum C: The microvesicle as a vehicle for EMMPRIN in tumor-stromal interactions. Oncogene 23: 956-963, 2004.

5. Garg K, Leitao MM Jr, Wynveen CA, et al: p53 overexpression in morphologically ambiguous endometrial carcinomas correlates with adverse clinical outcomes. Mod Pathol 23: 80-92, 2010.

6. Davidson B, Goldberg I, Berner A, et al: EMMPRIN (extracellular matrix metalloproteinase inducer) is a novel marker of poor outcome in serous ovarian carcinoma. Clin Exp Metastasis 20: 161-169, 2003.

7. Tsai WC, Chao YC, Sheu LF, et al: EMMPRIN and fascin overexpression associated with clinicopathologic parameters of pancreatobiliary adenocarcinoma in Chinese people. APMIS 115: 929-938, 2007.

8. Zheng HC, Takahashi $\mathrm{H}$, Murai $\mathrm{Y}$, et al: Upregulated EMMPRIN/CD147 might contribute to growth and angiogenesis of gastric carcinoma: a good marker for local invasion and prognosis. Br J Cancer 95: 1371-1378, 2006.

9. Zhong WD, Liang YX, Lin SX, et al: Expression of CD147 is associated with prostate cancer progression. Int J Cancer 130: 300-308, 2012.

10. Yu W, Liu J, Xiong X, et al: Expression of MMP9 and CD147 in invasive squamous cell carcinoma of the uterine cervix and their implication. Pathol Res Pract 205: 709-715, 2009. 
11. Klein CA, Seidl S, Petat-Dutter K, et al: Combined transcriptome and genome analysis of single micrometastatic cells. Nat Biotechnol 20: 387-392, 2002.

12. Wu W, Wang R, Liu H, et al: Prediction of prognosis in gallbladder carcinoma by CD147 and MMP-2 immunohistochemistry. Med Oncol 26: 117-123, 2009.

13. Morris PC, Anderson JR, Anderson B and Buller RE: Steroid hormone receptor content and lymph node status in endometrial cancer. Gynecol Oncol 56: 406-411, 1995.

14. Kleine W, Maier T, Geyer H and Pfleiderer A: Estrogen and progesterone receptors in endometrial cancer and their prognostic relevance. Gynecol Oncol 38: 59-65, 1990.

15. Pertschuk LP, Masood S, Simone J, et al: Estrogen receptor immunocytochemistry in endometrial carcinoma: a prognostic marker for survival. Gynecol Oncol 63: 28-33, 1996.

16. Deen S: FIGO staging of endometrial adenocarcinoma: a critical review and proposal. Int J Gynecol Pathol 28: 477-478, 2009.

17. Horn LC, Schmidt D, Fathke C and Ulrich U; Mitglieder der Organgruppe Uterus der AGO: New FIGO staging for uterine sarcomas. Pathologe 30: 302-303, 2009 (In German).

18. Kamat AA, Fletcher M, Gruman LM, et al: The clinical relevance of stromal matrix metalloproteinase expression in ovarian cancer. Clin Cancer Res 12: 1707-1714, 2006.

19. Gou XX, Jin F, Chen HX, Wu WL, Chen L and Zeng Y: Expressions of CD147, MMP-2 and MMP-9 in laryngea carcinoma and clinical significance. Zhonghua Yi Xue Za Zhi 90: 1264-1267, 2010 (In Chinese)

20. Wang S, Li B, Wang S, Li Y and Li J: Expression and clinical significance of CD147 and MMP-2 in squamous cell carcinoma and adenocarcinoma of the lungs. Zhongguo Fei Ai Za Zhi 14: 710-714, 2011 (In Chinese)

21. Tan $\mathrm{H}$, Ye K, Wang Z and Tang $\mathrm{H}$ : Clinicopathologic evaluation of immunohistochemical CD147 and MMP-2 expression in differentiated thyroid carcinoma. Jpn J Clin Oncol 38: 528-533, 2008.

22. Kanekura T and Chen X: CD147/basigin promotes progression of malignant melanoma and other cancers. J Dermatol Sci 57: $149-154,2010$

23. Xue YJ, Lu Q and Sun ZX: CD147 overexpression is a prognostic factor and a potential therapeutic target in bladder cancer. Med Oncol 28: 1363-1372, 2011

24. Rosenthal EL, Shreenivas S, Peters GE, et al: Expression of extracellular matrix metalloprotease inducer in laryngeal squamous cell carcinoma. Laryngoscope 113: 1406-1410, 2003.
25. Tan H, Ye K, Wang Z and Tang H: CD147 expression as a significant prognostic factor in differentiated thyroid carcinoma. Transl Res 152: 143-149, 2008.

26. Zhao L, Yu N, Guo T, et al: Tissue biomarkers for prognosis of prostate cancer: a systematic review and meta-analysis. Cancer Epidemiol Biomarkers Prev 23: 1047-1054, 2014.

27. Yang JM, Xu Z, Wu H, Zhu H, Wu X and Hait WN: Overexpression of extracellular matrix metalloproteinase inducer in multidrug resistant cancer cells. Mol Cancer Res 1: 420-427, 2003.

28. Liang Q, Xiong H, Gao G, et al: Inhibition of basigin expression in glioblastoma cell line via antisense RNA reduces tumor cell invasion and angiogenesis. Cancer Biol Ther 4: 759-762, 2005.

29. Tang Y, Nakada MT, Rafferty P, et al: Regulation of vascular endothelial growth factor expression by EMMPRIN via the PI3K-Akt signaling pathway. Mol Cancer Res 4: 371-377, 2006.

30. Yan L, Zucker S and Toole BP: Roles of the multifunctional glycoprotein, emmprin (basigin; CD147), in tumour progression. Thromb Haemost 93: 199-204, 2005.

31. Aristizabal P, Graesslin O, Barranger E, et al: A suggested modification to FIGO stage I endometrial cancer. Gynecol Oncol 133: 192-196, 2014.

32. Aliagas E, Vidal A, Texidó L, et al: High expression of ecto-nucleotidases CD39 and CD73 in human endometrial tumors. Mediators Inflamm 2014: 509027, 2014

33. Nakamura K, Kodama J, Hongo A and Hiramatsu Y: Role of emmprin in endometrial cancer. BMC Cancer 12: 191, 2012.

34. Ueda K, Yamada K, Urashima M, et al: Association of extracellular matrix metalloproteinase inducer in endometrial carcinoma with patient outcomes and clinicopathogenesis using monoclonal antibody 12C3. Oncol Rep 17: 731-735, 2007.

35. Morris PC, Anderson JR, Anderson B and Buller RE: Steroid hormone receptor content and lymph node status in endometrial cancer. Gynecol Oncol 56: 406-411, 1995.

36. Thigpen JT, Brady MF, Alvarez RD, et al: Oral medroxyprogesterone acetate in the treatment of advanced or recurrent endometrial carcinoma: a dose-response study by the Gynecologic Oncology Group. J Clin Oncol 17: 1736-1744, 1999. 